\title{
Multiple-Models Juxtaposition and Trade-offs among Modeling Desiderata
}

\begin{abstract}
This paper offers a characterization of what I call multiple-models juxtaposition (MMJ), a strategy for managing trade-offs among modeling desiderata. MMJ displays models of distinct phenomena together and fulfills different desiderata both in the individual models and by a comparison of those models. I discuss a concrete case from developmental biology, where MMJ coordinates generality and detail. I also clarify the distinction between MMJ and multiplemodels idealization (MMI), which also uses multiple models to manage trade-offs among desiderata. MMJ and MMI differ in several points, such as the ways they manage trade-offs and the purposes of using multiple models.
\end{abstract}

Yoshinari Yoshida

yoshi077@umn.edu

Department of Philosophy, Minnesota Center for Philosophy of Science, University of Minnesota, 831 Heller Hall, 271 19th Ave S, Minneapolis, MN 55455-0310, USA

\section{Acknowledgments}

I am grateful to Alan Love, Max Dresow, Katherine Liu, Michael Travisano, Samuel Fletcher, Michael Bennett McNulty, Christopher Nagel, Kelle Dhein, Aaron Vesey, and Nathan Lackey for their helpful comments on earlier drafts. Financial support for the research and writing of this paper came from Baruch Spinoza travel award, the International Society for the History, Philosophy, and Social Studies of Biology, and the Department of Philosophy at the University of Minnesota. Conversation with William Bausman helped me revise the paper. I would also like to thank the two anonymous reviewers for their useful suggestions. 


\section{Introduction}

The formulation of scientific models is often constrained by trade-offs among modeling desiderata, i.e., qualities that are valuable to scientists, but which may or may not be exhibited by a particular model. A classical discussion of this issue is Levins's (1966) analysis of model building in population biology. Levins describes the difficulties involved in formulating a model that is maximally general, realistic, and precise at the same time. Although generality (applicability of a model to a wide range of things), realism (faithfulness of a model as a representation of the target), and precision (regarding predictions made by a model about the target) are all valuable, population biologists cannot build a single model that maximizes all three desiderata simultaneously. As a consequence, they typically sacrifice one desideratum to make a model with one or more of the other desiderata. For instance, one approach focuses on particular cases of the phenomenon of interest and makes a model based on accurate measurements; the resulting model can produce precise and realistic predictions, but its applicability is narrow. Another approach makes an unrealistic model that ignores parameters like time lags and physiological states, but is generally applicable and can produce precise predictions (Levins 1966).

It is debatable whether the exact trade-off relationship that Levins describes really holds. Orzack and Sober (1993) take Levins to be discussing a trade-off derived from properties of the formalism used in population biology and argue that there is no such trade-off relationship among generality, realism, and precision. Odenbaugh (2003) disagrees with Orzack and Sober and argues that the trade-off does exist in the sense that pragmatic constraints involved in population biologists' model building do not allow the simultaneous fulfillment of those desiderata in a single model. Despite such disagreement over interpretation and evaluation of the 
detail of Levins's account, philosophers nonetheless agree that its core insight is right and useful; that is, in model building and evaluation in science, some desiderata exist in trade-off relationships and hence cannot be simultaneously maximized or increased in a single model (Gelfert 2013; Matthewson 2011; Matthewson and Weisberg 2009; Odenbaugh 2003; Weisberg 2004).

How do scientists manage such trade-offs? As I already mentioned, a strategy that Levins advocates is to formulate more than one model to address a phenomenon. Weisberg $(2007,2013)$ develops this idea and calls the strategy multiple-models idealization (MMI). MMI makes use of multiple models that idealize the phenomenon of interest differently. Those models prioritize different desiderata and collectively help scientists achieve their goals (explanation, prediction, understanding, control, etc.). MMI is used most often where researchers are investigating highly complex phenomena.

Although MMI is an important strategy that is adopted in various contexts in science, it is not the only strategy available to manage trade-offs among modeling desiderata. This paper discusses another strategy, which also involves multiple models but is distinct from MMI. In scientific practice, models of multiple related phenomena are sometimes displayed together in a representation. This presentational practice facilitates the fulfillment of certain desiderata through comparison of those models, while maintaining desiderata that the individual models exemplify. Consequently, desiderata that a single model cannot exhibit simultaneously can be fulfilled in the set of juxtaposed models. I call this strategy multiple-models juxtaposition (MMJ).

To illustrate how MMJ works, I conduct a case study that focuses on investigations of branching morphogenesis in developmental biology. Mechanistic models of the formation of 
different branched organs are often displayed together and compared in review articles.

Moreover, the particular mechanistic models that are compared have changed over time in the field. I argue that we can understand this presentational practice as a case of MMJ that coordinates a trade-off between the desiderata of generality and detail. Although researchers of branching morphogenesis are interested in both providing detailed descriptions of individual branching mechanisms and finding general features of them, these are often in a trade-off relationship for a single model. By displaying multiple models together and comparing them, researchers provide generalizations of features shared across the distinct mechanisms while keeping detailed descriptions of those mechanisms in the individual models. The shift from one comparison to another over time can also be understood in terms of modeling desiderata. It reflects the preference for generalizing certain features of the mechanisms over another. Thus, we can account for this case by adopting the idea of MMJ.

The next section discusses generality and detail as modeling desiderata and the trade-off between them, especially as manifested in the context of mechanistic explanation. Then I turn to the case study. As a part of this case study, I describe a shift from one comparison of mechanisms to another over time with a special focus on what commonalities were highlighted in each comparison. Section 4 asks three questions about the case: why do the researchers of branching morphogenesis often display multiple models together?; why has one comparison become more common than the other?; and why don't the researchers make a single unified model instead of formulating multiple models and displaying them together? Answers to those questions illustrate how MMJ manages the trade-off between generality and detail. Section 5 compares MMJ with MMI. Despite their apparent similarity, these strategies have several 
contrasting features. This comparison leads us to a deeper understanding of MMJ, as well as promoting new inquiry into MMI.

\section{Generality and Detail}

Generality and detail are often (but not always) in a trade-off relationship. Generality is a desideratum shared in many fields and contexts of science. A model is general when it is applicable to a wide range of things (Matthewson 2018; Matthewson and Weisberg 2009). I use an inclusive expression ("a wide range of things") because different sciences have different domains of inquiry. Even within biology, generalizations can hold over various domains, such as geographical regions, taxa, cell lineages, spatial parts of an organism, and periods of time, depending on which subfield of biology a generalization belongs to (Waters 1998). Here I focus on developmental biology, where generality of a model is often understood in terms of taxa, component systems of an organism, developmental stages, and/or spatial scales. "The induction of numerous organs is effected by a relatively small set of paracrine factors. The embryo inherits a rather compact genetic "tool kit" and uses many of the same proteins to construct the heart, kidneys, teeth, eyes, and other organs. Moreover, the same proteins are used throughout the animal kingdom - the factors active in creating the Drosophila eye or heart are very similar to those used in generating mammalian organs" (Gilbert 2014, 84). This passage emphasizes that a signaling mechanism often accounts for developmental phenomena across component systems (e.g., "heart, kidneys, teeth, eyes, and other organs") as well as across taxa (e.g., "throughout the animal kingdom").

The desideratum of detail consists in the inclusion of relevant features of the target system in the model. A detailed model provides substantial information about component 
features of the system it represents. Although detail might appear similar to realism, these are distinct desiderata. Realism is the faithfulness of a model as a representation of a target system, and this faithfulness depends on the extent to which false assumptions or idealizations are not included in a model. The more realistic a model is, the less false assumptions it will have (i.e., the less idealized it is). On the other hand, detail concerns the extent to which relevant features of the target system are included in a model. The more detailed a model is, the less features of the target system it will ignore (i.e., the less abstract it is). ${ }^{1}$

Detail plays an important role in mechanistic explanation. A mechanistic model is often detailed in a specific way. According to a philosophical formulation called minimal mechanism, a "mechanism for a phenomenon consists of entities (or parts) whose activities and interactions are organized so as to be responsible for the phenomenon" (Glennan and Illari 2018, 2). Hence, a model of a process underlying a phenomenon is considered mechanistic when it provides relevant details about what entities are involved, what those entities do, and how those activities and interactions are organized to bring about the phenomenon. These details are often represented in the form of diagrams, where visual representations facilitate the reasoning of and communication among researchers (Abrahamsen and Bechtel 2014; Abrahamsen et al. 2018; Sheredos et al. 2013). It is important to note that "detail is a desideratum" does not mean that more detailed models are always preferable. What degree of detail is appropriate depends heavily on the communities, contexts of research, and questions being asked. Similarly, what types of

\footnotetext{
${ }^{1}$ Throughout this paper, I use the term "abstraction" as a property of representations. It means omission or ignorance of detail in representations and has nothing to do with abstract entities (see Levy and Bechtel 2013).
} 
relevant features must be included in the model can vary depending on the situation (see Bechtel 2017; Levy and Bechtel 2013).

Generality and detail are often in a trade-off relationship. This relationship is illustrated by the following example. A simple model of a cell in which only the cell membrane and nucleus are depicted is applicable to most eukaryotic cells. This is because characteristic features of different types of eukaryotic cells are abstracted away from the representation. But if the model includes an axon and dendrites, then it becomes less general; the model is applicable only to neurons. If more characteristic features of a specific type of neuron are added to the model, its generality decreases. The more detailed a representation is, the less general it is likely to be.

This trade-off is an obstacle when both generality and detail are pursued. We can find examples of such situations in many life sciences, where describing mechanisms in detail is a common way of explaining biological phenomena, although many researchers also seek widely applicable models. The simplest response to this trade-off is to seek a balance between generality and detail in a single model. There are biological processes that can be regarded as occurring in a stereotypical manner when they are modeled at the level of detail appropriate for the purposes of research. For instance, a simple model of synaptic transmission in an introductory biology textbook is sufficiently general as well as sufficiently detailed because the model, which describes the transmission mechanism in a degree of detail appropriate for a novice, is applicable to a broad range of instances (i.e., different types of neurons in different species). Such reconciliation of generality and detail in a single model is a matter of widespread importance in the formulation of mechanistic models, as well as in many other fields and contexts in science. However, it is not always effective. Phenomena that scientists hope to explain are often patterned while also being variable, in particular in the life sciences. It is often the case that an interesting 
feature is shared in a range of processes, whereas there are also non-negligible dissimilarities among them. In such cases, if one formulates a model with enough detail for the purposes of research, generality of the model may substantially decrease; that is, detail and generality of an appropriate degree cannot be reconciled in a single model.

Matthewson (2018) calls attention to a different way to reconcile generality and detail in a single model. This representational strategy describes a causal interaction in a relatively abstract way, while inserting in the representation a component mechanism of the interaction as an inset. This inset provides information about the detail of the mechanism that is a part of the causal interaction in specific cases. This does not impair the generality of the entire model because one can recognize the abstract pattern in the whole causal interaction and understand that that pattern is applicable to various phenomena, even if the inserted component mechanism occurs only in a subset of them. Another strategy that can coordinate the trade-off between generality and detail is MMI. One can fulfill both desiderata by constructing an abstract model of a phenomenon that is generally applicable to many instances and a detailed model of the same phenomenon that has narrower applicability. For example, whereas chronobiologists build a detailed model of a gene regulation mechanism underlying certain periodic behavior that includes specific types of genes and proteins, they also construct an abstract model of the same mechanism that ignores specific types of entities and focuses on its organization (e.g., the causal connectivity of the mechanism). The former model provides detailed description of specific cases, while the latter model enables an explanation that can be applied to a wide range of cases (Bechtel 2017; Levy and Bechtel 2013).

MMJ coordinates generality and detail in a different way from any of these strategies. It involves multiple models about related but distinct phenomena and provides a detailed 
description of a causal process in each model and a generalization of shared features through a comparison of the multiple models. Let us move on to a concrete case to see how this strategy works in scientific practice.

\section{Case Study: Research on Branching Morphogenesis}

My case study is taken from research on branching morphogenesis in developmental biology. Branching morphogenesis is a set of processes by which branched structures in organs (e.g., blood vessels, kidneys, lungs, and mammary glands) are formed through various cellular behaviors in biological development. Developmental biologists have studied cellular and molecular mechanisms that produce such branched structures. They have been interested not only in how each branched structure is made, but also in how similar the mechanisms are across species and across organs (and sometimes across developmental stages and across spatial scales). This dual interest in generality and detail is expressed most typically in review articles, where the authors seek to provide detailed descriptions of individual branching mechanisms as well as discuss applicability of and similarity between those mechanisms (e.g., Affolter et al. 2003, 2009; Davies 2002; Ochoa-Espinosa and Affolter 2012; Spurlin and Nelson 2017; Varner and Nelson 2014).

I focus on two comparisons of mechanistic models of branching morphogenesis, each of which is between models of two mechanisms that operate in different biological systems. Around 2000, researchers often compared the mechanism for fly tracheal formation with the mechanism 
for mammalian lung formation. ${ }^{2}$ In this comparison, shared molecular types involved in both mechanisms were highlighted. I call this Comparison 1. Over the last decade, however, it has become more common for review articles to compare the mechanism for fly tracheal formation with the mechanism for vertebrate angiogenesis. ${ }^{3}$ This new comparison emphasizes shared cellular activities and overall organization, instead of molecular types that trigger morphogenesis. I call this Comparison 2.

I selected this example because it exemplifies clearly how MMJ functions to manage a trade-off between modeling desiderata. Importantly, what the case illustrates is not peculiar to developmental biology. Pursuit of both generality and detail in representations is common in many fields of life science, such as molecular biology, cell biology, physiology, and neuroscience, and researchers in these fields often display models of distinct phenomena together to achieve these kinds of comparisons (e.g., Fontana et al. 2010, figure 3; Harmer et al. 2001, figure 2; Ryan and Grant 2009; cf. Abrahamsen et al. 2018; Bechtel 2009). Hence, although I concentrate on a single example, the pattern of practice that I describe and the philosophical insights derived from it have a wide range of application. Indeed, MMJ may be used even outside of the life sciences, though I leave this as a topic for future research.

\footnotetext{
${ }^{2}$ The tracheal system of insects consists of ramifying epithelial tubes that directly transport the air to tissues throughout the body for gas exchange.

${ }^{3}$ Angiogenesis refers to blood vessel formation through branching from preexisting blood vessels.
} 
As we saw in the previous section, a mechanistic model provides information about relevant entities, their activities, and the overall organization of those activities. When mechanistic models are compared, it becomes important to determine how many of these components are similar and how similar those components are (Love 2018). Note that we are here talking about the comparison of mechanistic models in general, not about the judgment of the evolutionary conservation of mechanisms. Although evolutionary conservation is an important reason why we find similar mechanisms in different living systems (Bechtel 2009; Halina and Bechtel 2013), the comparison of mechanistic models precedes the judgment of evolutionary conservation and can be seen as a distinct activity.

\subsection{Comparison 1—Shared Signaling Molecule}

In 1996, it was reported that a newly found fly homolog of the fibroblast growth factor (FGF), which was named Branchless (Bnl), is required for tracheal formation in fruit flies (Sutherland et al. 1996). ${ }^{4} \mathrm{Bnl}$ is a protein secreted by the tissue surrounding the trachea. It guides branching of the trachea by attracting cells at the leading tip of the developing tubes. Following this finding, evidence was provided that FGF-10 is secreted in mesenchymal tissue surrounding the developing epithelial tubes in the mouse lung and that FGF-10 guides the directional growth and controls branching of the lung (Bellusci et al. 1997; Min et al. 1998; Park et al. 1998; Sekine et al. 1999). Some of the studies pointed out the similarity between the mechanism for fly tracheal formation and the mechanism for mouse lung formation. "[I]n both Drosophila and

\footnotetext{
${ }^{4}$ FGFs are a family of growth factors that are known to regulate various developmental processes.
} 
mouse the production of a branched respiratory system involves the directional movement of respiratory cell precursors towards a localized source of an FGF ligand, either by migration and elongation, or by outgrowth of epithelial buds" (Bellusci et al. 1997, 4876). In this comparison, the most crucial similarity between the two mechanisms was the involvement of the same kind of molecule, i.e., FGF. FGF molecules (Bnl in fly trachea and FGF-10 in mouse lung), which are secreted locally in nearby tissues, guide the directional outgrowth of the tubes by stimulating their extension. However, there were also important differences. For example, cellular activities underlying tube extension appeared to be different. In the fly tracheal formation, cells that experience a high concentration of FGF become migratory and move towards the source of FGF. In contrast, it was known that cell migration is not the driving force of the directional outgrowth during mouse lung development (Nogawa et al. 1998). ${ }^{5}$

A review article that compared the two mechanisms displayed diagrams of these mechanisms together (Metzger and Krasnow 1999) (figure 1). These diagrams highlight the shared type of signaling molecule (FGF; blue points in the figure) and its role of guiding the extension of the tubes. The figure also showed some differences, such as cellular activities and overall organization. The difference in cellular activities is indicated in the right diagrams; fly

${ }^{5}$ Cellular activities during mouse lung development is less understood even today. It was suggested that branching in the lung is based on differential proliferation. The idea was that pulmonary cells that experience a high concentration of FGF become more proliferative, which causes the directional outgrowth (Bellusci et al. 1997). However, a later study suggested that the formation of pulmonary outgrowth is not likely to be based on differential proliferation (Nogawa et al. 1998). 
tracheal cells at the tip of the tube extend filopodia for migration, whereas mouse lung cells are not migratory and maintain the smooth, sheet-like structure throughout the tube. ${ }^{6}$ This and the difference in the numbers of tubular cells involved in each outgrowth make the overall organization of these mechanisms quite different.

\section{INSERT FIGURE 1 HERE}

Therefore, Comparison 1 accentuated the common type of secreted signaling molecules (FGF). It also involved the difference in cellular activities (tube extension based on directed migration vs. non-migratory extension), which made the overall organization of the mechanisms dissimilar as well.

\subsection{Comparison 2-Shared Cellular Activities and Organization}

An understanding of vertebrate angiogenesis at the molecular level developed several years after the basic mechanisms for fly tracheal formation and mouse lung formation were elucidated. It was demonstrated that vascular endothelial growth factor A (VEGF-A) secreted from a nearby tissue guides angiogenic sprouting by promoting filopodia extension from the cells at the tip of angiogenic vasculature ${ }^{7}$; VEGF-A also promotes proliferation of cells located at the stalk of the blood vessels (the former are called "tip cells," while the latter are called "stalk cells") (Gerhardt et al. 2003). Studies published several years later revealed that Notch signaling

\footnotetext{
${ }^{6}$ Filopodia are long and thin cytoplasmic projections that play important roles in cell migration. ${ }^{7}$ VEGFs are a family of growth factors that are known for their roles in the development and maintenance of blood and lymphatic vessels.
} 
is involved in the specification of tip and stalk cells ${ }^{8}$; vascular cells that experience a high concentration of VEGF acquire the tip cell phenotype and prevent neighboring cells from becoming tip cells by lateral inhibition through Notch signaling (Hellström et al. 2007; Siekmann and Lawson 2007). Then, stalk cells push the tip cells by elongating the tube through proliferation and rearrangement. These studies, along with further studies of fly tracheal formation, revealed interesting similarities between the mechanisms for fly tracheal formation and for vertebrate angiogenesis. For example, it had been shown that determination of migratory cells and other cells in the fly trachea is also based on lateral inhibition through Notch signaling (Llimargas 1999; Ghabrial and Krasnow 2006). As a result, over the last decade, researchers of branching morphogenesis have focused more on Comparison 2, rather than Comparison 1. It has become common for review articles to display the mechanistic models of fly tracheal formation and vertebrate angiogenesis (instead of fly tracheal formation and mammalian lung formation). Some authors explicitly mention the shift and emphasize the similarity between the mechanisms compared in Comparison 2: "For many years, tracheal branching has been compared most often to mammalian lung branching because both organs are involved in oxygen transport and because branching of both organs is controlled by FGF signalling ... However, recent studies have unravelled unexpected and stunning similarities in cellular behaviour between tracheal branching in D. melanogaster [fruit fly] and angiogenic sprouting in vertebrates" (Affolter et al. 2009, 833).

The mechanisms compared in Comparison 2 have different features in common from those compared in Comparison 1. What is salient is the similarity of cellular activities. During

\footnotetext{
${ }^{8}$ Notch signaling is a signaling pathway that works between cells in contact and plays crucial roles in various developmental processes.
} 
both fly tracheal formation and vertebrate angiogenesis, tubular cells that experience high concentrations of the signaling molecules become tip cells; tip cells migrate towards the source of the signaling molecules; tip cells prevent neighboring cells from becoming tip cells by lateral inhibition through Notch signaling; and those neighboring cells become stalk cells, which collectively push the tip cells. (Tip cells and stalk cells are defined in terms of the functions they play during branching morphogenesis, and these terms are now used for describing both mechanisms.) Because of these similarities, the overall organization of the mechanisms is very similar as well. However, there are also several non-negligible differences. The most significant difference is the types of secreted signaling molecules that guide tip cell migration. FGF plays this role in the fly trachea, whereas VEGF accomplishes it during vertebrate angiogenesis, and FGF and VEGF are not very closely related. There is also a difference in cellular activities. Stalk cells push the tip cells by intercalation during fly tracheal formation, whereas during vertebrate angiogenesis, the pushing force of stalk cells is based on their proliferation and rearrangement.

Figure 2 shows some examples of the display of the two mechanistic models together, all of which are from review articles about branching morphogenesis (Ochoa-Espinosa and Affolter 2012; Spurlin and Nelson 2017; Wang et al. 2017). They reveal the cellular activities and overall organization shared between the two mechanisms. The functional difference between tip and stalk cells (tip cells migrate towards the source of the diffusing ligands, whereas stalk cells push the tip cells and extend the outgrowth) is highlighted by distinct colors or text within the diagrams. The interaction between tip and stalk cells-lateral inhibition through Notch signaling - is also clearly shown in all figures by flat-edged arrows and text (a flat-edged arrow is the convention to represent an inhibitory influence). These mechanism diagrams are displayed with very similar compositions, which emphasizes the similarity in overall organization of the 
two mechanisms. Each pair of mechanistic models also shows differences between the two mechanisms. For example, they indicate by text within the diagrams the different types of proteins (Bnl/FGF in fly tracheal formation; VEGF in vertebrate angiogenesis) that play the role of attracting tip cells. They also show the difference in how stalk cells push tip cells (intercalation during fly tracheal formation; proliferation and rearrangement during vertebrate angiogenesis) visually and textually.

\section{INSERT FIGURE 2 HERE}

Comparison 2 can be summarized as follows. It highlights the commonality of some cellular activities and overall organization (directed migration, lateral inhibition, pushing of tip cells), as well as signaling that mediates the tip-stalk interaction (Notch signaling). There are also differences, such as types of secreted signaling proteins (FGF vs. VEGF) and some cellular activities (intercalation vs. proliferation and rearrangement).

\section{Modeling Desiderata and Presentational Choice}

This section asks three questions about the cases introduced in the previous section: (a) why do the researchers of branching morphogenesis - in particular, those writing about Comparison 2- display multiple mechanistic models together?; (b) why has Comparison 2 become more common than Comparison 1?; and (c) why don't the researchers of branching morphogenesis make a single unified model instead of formulating multiple models and presenting them together? This section answers these questions by focusing on the two desiderata, generality and detail, and the trade-off between them.

First, why do the researchers of branching morphogenesis display multiple models together? A short answer to this question is: to highlight features shared across different 
branching mechanisms. This reflects the desideratum of generality. Researchers of branching morphogenesis are interested in which pairs (or groups) of mechanisms are more similar than others. Take Comparison 2, for example. The two mechanistic models are displayed together so that the similarities between them are easily recognized. The same terms, i.e., "tip cell" and "stalk cell," are used to characterize both mechanisms. The similar roles these types of cells play are made evident by the use of distinct colors, text, or arrows in the diagrams. The similar compositions of the diagrams also facilitate recognition of the resemblance in overall organization. Through these forms of representation, those diagrams highlight general featurescellular activities and overall organization — of the two mechanisms. ${ }^{9}$ Importantly, the generalization highlighted in Comparison 2 has influenced research on branching morphogenesis and related areas. For instance, reflecting on the similarity between the mechanisms for fly tracheal formation and vertebrate angiogenesis, Munóz-Chápuli (2011) asks why they are so similar and proposes a hypothesis that they have evolved by adopting a conserved hypoxiaresponse mechanism. Another example is the suggestion that fly tracheal system might serve as a useful model of tumor angiogenesis because of the known similarity between the mechanisms for tracheal formation and angiogenesis (e.g., Murray 2015). These examples show that

${ }^{9}$ Generality alone is not the only answer to why researchers display multiple models together because we can ask further why the strategy of MMJ is adopted instead of another strategy for generalization. A fuller answer is provided in my response to the last question, which is about why researchers do not produce a single, unified model. 
Comparison 2 is more than just a convenient way to summarize different branching mechanisms; it sometimes influences directions of research. ${ }^{10}$

Note that each of the mechanistic models being compared is by itself a generalization. For example, the mechanism for vertebrate angiogenesis underlies the development of different blood vessels (e.g., retinal blood vessels and intersegmental arteries) in different species (e.g., mouse and zebrafish) at different developmental stages (e.g., embryonic and adult) (Siekmann et al. 2008). However, by presenting the model of this mechanism together with the model of the mechanism for fly tracheal formation, one can capture features common across these mechanisms. The shared cellular activities and overall organization highlighted by this comparison are more general than those of each mechanism.

Next, why has Comparison 2 become more common than Comparison 1 ? This shift occurred in part because the studies showed that the mechanism for vertebrate angiogenesis is similar in many respects to the mechanism for fly tracheal formation. However, this is not enough as an answer because we cannot say that the mechanism for vertebrate angiogenesis is more similar to the mechanism for fly tracheal formation simpliciter than to the mechanism for

10 These cases also suggest that there are multiple reasons why scientists pursue generality. Generalizations sometimes serve to identify objects for further investigations (e.g., evolutionary basis of the similarity between the different developmental mechanisms). In other cases, they help in the search for useful models to study systems of particular interest (e.g., fly trachea as a model of tumor angiogenesis). We should understand the value of generality for scientists as consisting of both its intrinsic value and usefulness for other purposes. 
mammalian lung formation. Recall that although Comparison 2 highlights cellular activities and overall organization shared across the two mechanisms, it also involves differences, such as in types of signaling molecules that guide the directional outgrowth. Recall also that Comparison 1 captures exactly the latter kind of feature, i.e., the type of signaling molecule, which is shared across the mechanisms for fly tracheal formation and mammalian lung formation. We cannot judge which pair of mechanisms are more similar without having decided what kind of features of those mechanisms are of interest. Therefore, to have a deeper answer to the question of why Comparison 2 is now more common than Comparison 1, we must further ask: given the known facts about these three mechanisms, why has Comparison 2 (which focuses on the shared cellular activities and overall organization) become more common than Comparison 1 (which focuses on the shared type of signaling molecule)?

To answer this question, we have to consider two subclasses of the desideratum of detail: cellular detail and molecular detail. Cells and molecules are two major types of entities that constitute developmental mechanisms. A model with more cellular detail provides more information about cellular features of the target phenomenon, such as what kind of cells are involved and how those cells act and interact. Similarly, a model with more molecular detail involves more information about molecular features of the target. Within the field of developmental biology, how these desiderata are treated vary depending on subcommunities, contexts of research, and questions being asked. Where only cellular detail is pursued, researchers formulate models that focus on cellular features and include very little information about molecular features. In other situations, mechanistic models that focus on molecular detail are formulated (Love 2018). There are also situations where both desiderata are pursued, as is the 
case for Comparison 1 and 2. Even in the latter type of situation, however, one desideratum can be prioritized over the other.

I argue that Comparison 2 has become more common than Comparison 1 because cellular detail is prioritized in the studies of branching morphogenesis. Branching morphogenesis falls under the category of morphogenesis. Morphogenesis is characterized as a set of processes by which three-dimensional biological structures are formed through various kinds of cellular behaviors. Thus, cellular features and dynamics have been regarded as a key component of morphogenesis (e.g., Trinkaus 1969). Consider, for example, the following passage from Scott Gilbert's Developmental Biology (a well-known textbook in the field): "During development, cells divide, migrate, and die; tissues fold and separate. Our fingers are always at the tips of our hands, never in the middle; our eyes are always in our head, not in our toes or gut. This creation of ordered form is called morphogenesis, and it involves coordinating cell growth, cell migration, and cell death" (Gilbert 2014, 2; emphasis added). We can find a similar idea in older texts, for example: "The word 'morphogenesis' is often used in a broad sense to refer to many aspects of development, but when used strictly it should mean the moulding of cells and tissues into definite shapes" (Waddington 1956, 433; emphasis added).

To solve problems of branching morphogenesis involves articulating how various kinds of cellular behaviors (proliferation, migration, death etc.) produce three-dimensional branched structures. For those who have a strong interest in morphogenesis, in particular generalizations therein, Comparison 2 is preferable to Comparison 1 because the former generalizes cellular activities and organization. This explains why Comparison 2 has become more common over the last decade. In the late 1990's, when the detailed mechanism of vertebrate angiogenesis was not known, researchers were focusing on Comparison 1, which highlights the shared signaling 
molecule. However, once cellular aspects of vertebrate angiogenesis were revealed, attention has shifted to Comparison 2 because it fits their interest in (generalizing) cellular features. This does not necessarily mean that Comparison 2 is a better comparison simpliciter. It is possible that in different communities or contexts where generalizing molecular features is prioritized, Comparison 1 becomes more common. Also, it does not mean that researchers of branching morphogenesis are not interested in signaling molecules; molecular detail is also important for them (see below). What the claim means is that, with respect to the interest in common cellular behaviors, there is a good reason to prefer Comparison 2, since its focus of generalization pertains to cellular features.

Now consider the third question: why don't the researchers make a single unified model? If the researchers of branching morphogenesis want to generalize the cellular activities and overall organization common across the mechanisms for fly tracheal formation and vertebrate angiogenesis, there is a simpler way than displaying multiple models together. A single model that can explain both phenomena could be formulated by abstracting certain elements away from each model. Recall the differences between the two mechanisms in Comparison 2 (e.g., FGF vs. VEGF). By ignoring these differences, one could make a single model that is a little more abstract but is more general than both mechanistic models. Such a model would contain only the information about the common features between the two mechanisms; it would use generic expressions without specifying the differences (e.g., "signaling molecule" instead of "FGF" and "VEGF"). Nevertheless, many authors choose to display the two mechanistic models together instead of formulating such a unified abstract model. Why do they do this?

My answer is that it is because such an abstract model does not provide enough molecular detail and molecular detail is an important desideratum in developmental biology. 
Identifying molecules that trigger a developmental process has been regarded as a crucial step in articulating an explanation since the molecularization of the research of development (Burian and Thieffry 2000; Hopwood 2009). Because of this desideratum, the researchers of branching morphogenesis usually do not abstract away key signaling molecules from their representations. They adopt MMJ instead.

On the one hand, the researchers seek to formulate the general features of the different mechanisms for branching morphogenesis; in particular, they are interested in generalizing cellular activities and overall organization, which reflects the desiderata of generality and of cellular detail. On the other hand, the molecules that trigger the branching processes - FGF or VEGF in the present case - must not be ignored because of another desideratum shared widely in developmental biology, i.e., molecular detail. MMJ is adopted because it enables satisfying all of these desiderata simultaneously. The multiple models displayed together can represent cellular activities and overall organization shared across the two mechanisms for fly tracheal formation and vertebrate angiogenesis, while keeping the information about the signaling molecules, which differ between these mechanisms. MMJ serves to coordinate the desiderata that are in the tradeoff relationship.

Before proceeding to the next section, I defend my focus on MMJ against a potential objection. MMJ is a presentational strategy and concerns the summarization and communication of models in review articles, textbooks, and conference presentations. This might cause the reader to wonder whether MMJ is worthy of philosophical investigation. While the presentation of models might have practical import for scientists, does it have any distinct philosophical implications? I respond to this objection by appealing to Andrea Woody's (2004, 2014, 2015) functional approach to philosophy of science. For example, Woody (2014) uses the example of 
the explanatory roles of the periodic table to argue for the philosophical importance of representation. According to Woody, it is the specific representation (i.e., periodic table), rather than the abstract content being represented (i.e., periodic law), that has explanatory power in chemistry. To understand how the periodic law enables particular explanatory activities, we must look at the representational practice. This approach recognizes that scientists are practitioners with limited cognitive capacities and that science is a social enterprise (Woody 2014). What scientists can do with their models depends in part on how those models are presented and represented (e.g., Abrahamsen and Bechtel 2014; Abrahamsen et al. 2018; Sheredos et al. 2013). My case study makes an analogous point to Woody's argument by focusing on generalization instead of explanation. As I showed, the desideratum of generality is often fulfilled by comparisons of multiple models, which are facilitated by a specific form of presentation. If we simply look at the abstract content of individual models and ignore how they are presented and represented, then we would not understand what and how generalizations are embraced in the field. This in turn means that we would not understand how the trade-off between generality and detail is managed. Therefore, presentation and representation are essential to understand the justification of significant and widespread scientific reasoning activities.

\section{Multiple-Models Idealization and Multiple-Models Juxtaposition}

So far, this paper has focused on characterizing MMJ and analyzing how it functions. This section asks how and in what respects MMJ is different from MMI. MMI is characterized as a strategy to manage trade-offs by fulfilling different desiderata in different models (Weisberg 2007, 2013). This strategy is effective especially where scientists aim to account for a highly complex phenomenon. If the phenomenon of interest is highly complex, then no single model 
that is tractable to human beings can instantiate all of the modeling desiderata that scientists hope to fulfill. This problem can be dealt with by formulating multiple models with different desiderata. Weisberg highlights a few examples where MMI is adopted: ecologists' constructing multiple models to explain a phenomenon such as predation, chemists' reliance on both molecular orbital and valence bond models, and the use of multiple models of global circulation by the United States National Weather Service (NWS) to predict weather.

Both MMI and MMJ utilize multiple models to fulfill desiderata that a single model cannot exhibit simultaneously because of trade-offs among them. However, there are some contrasting features between the two strategies. The most crucial difference is the ways they manage trade-offs among modeling desiderata. MMI can fulfill desiderata that are in trade-off relationships because the multiple models it formulates are of different types (e.g., they are based on different idealization assumptions), and hence they can exhibit or maximize different desiderata. The point of MMI is that each model fulfills distinct desiderata. In contrast, the multiple models in MMJ are formulated and represented in the same way. In Comparison 1 and 2, for example, the models displayed together are both mechanistic models; they are depicted at the same resolution (i.e., in the same degree of detail) and adopt the same rules of representation (i.e., colors, arrows, and text are used consistently in the two models) (Figures 1-2). MMJ does not rely on different types of models to manage trade-offs. Instead, it avoids trade-offs by fulfilling certain desiderata through a comparison of models that are distinct from the desiderata exhibited by the individual models. The source of generality is a comparison of models, while detail is instantiated by the individual models.

Model comparison itself is not unique to MMJ. It plays a role also for MMI in what is called robustness analysis. Robustness analysis is a means to judge the reliability of predictions 
made by models (Levins, 1966; Weisberg, 2006, 2013; Wimsatt 2012). Most models involve some idealization and it is sometimes difficult to determine which features of a model reflect properties of the target phenomenon and which features are attributed to idealization assumptions peculiar to the model. A comparison of multiple models facilitates this judgment. If those models make a common prediction, even though they are based on different idealization assumptions, then that prediction is robust and thus likely to capture a genuine feature of the target phenomenon. There is a crucial difference between robustness analysis in MMI and model comparison in MMJ. Although multiple models are compared to identify features common across them in both cases, these are epistemically distinct activities. Robustness analysis is aimed at evaluating how reliable the predictions made by the individual models are. It compares the models to identify components of those models that are likely to reflect genuine features of the target phenomenon (Weisberg 2013). Model comparison in MMJ is not aimed at such an evaluation of the components of individual models, but at fulfilling certain desiderata. The reliability of aspects of the individual models is not at stake. Displaying multiple models together in MMJ tells us nothing about which components of those models are reliable. Instead, it highlights features shared across the target phenomena in a way that we can easily recognize them.

This difference in the use of model comparison suggests another difference about the purposes of using multiple models. In MMI, multiple models are formulated to account for a broad phenomenon from different perspectives. The point of the multiplicity of models in MMI is that they contribute in different ways to the understanding of the same phenomenon. (Note that these multiple models need not and often do not represent exactly the same target phenomenon; they are used to address the same phenomenon in MMI.) In contrast, the purpose of using 
multiple models in MMJ is to compare multiple distinct phenomena by comparing models of them. Here the multiplicity of models reflects the multiplicity of the target phenomena. For example, fly tracheal formation and vertebrate angiogenesis are distinct subclasses of branching morphogenesis. The multiple mechanistic models are formulated to compare them efficiently.

Table 1 is a summary of the contrasting features of MMI and MMJ. MMI is an idealization strategy to manage trade-offs among modeling desiderata by formulating multiple models on the basis of different idealization assumptions so that those models exhibit different (combinations of) desiderata. It utilizes the multiple models to better account for a single phenomenon. A comparison of models is not essential to MMI, but it is sometimes conducted for robustness analysis, where models are compared to judge which components of them are likely to reflect genuine features of the target phenomenon. MMJ is a presentational strategy to manage trade-offs by fulfilling modeling desiderata both in individual models and by a comparison of those models. Model comparison is essential to MMJ. It displays multiple models of the same type that represent distinct phenomena to facilitate a comparison between them. Although I have focused on dissimilarities between MMI and MMJ, the purpose of this section was to contrast the two strategies in order both to provide a more detailed characterization of MMJ and to identify potential interesting issues concerning MMI that have not been analyzed in depth. By comparing the two strategies and trying to articulate how and in what respects they are different, we can acquire a deeper understanding of both of them.

\section{INSERT TABLE 1 HERE}

\section{Concluding Remarks}


This paper characterized multiple-models juxtaposition (MMJ) as a strategy for managing trade-offs among different modeling desiderata. MMJ displays models of similar but distinct phenomena together and fulfills desiderata that are in trade-off relationships in the individual models and by a comparison of those models. This point is illustrated by the case study of branching morphogenesis, where mechanistic models of the formation of different branched structures are frequently displayed together in a representation. I focused on two desiderata that are important for this area of research, generality and detail, and showed that MMJ is adopted to manage the trade-off between them. I also compared MMJ with MMI. Although both strategies use multiple models to fulfill desiderata in trade-off relationships simultaneously, there are interesting differences, such as the ways they manage trade-offs, the types of models they involve, how they use model comparison, and the purposes of using multiple models. This comparison helps us better understand the scientific practice of MMJ, as well as MMI, and suggests that further inquiry is needed to understand the diverse ways that scientists fulfill a variety of modeling desiderata.

\section{References}

Abrahamsen, Adele, and William Bechtel. 2014. "Diagrams as Tools for Scientific Reasoning." Review of Philosonhv and Psvchologv 6 (1): 117-31.

Abrahamsen, Adele, Benjamin Sheredos, and William Bechtel. 2018. "Explaining Visually Using Mechanism Diagrams." In The Routledge Handbook of Mechanisms and Mechanical Philosophy, edited by Stuart S. Glennan and Phyllis Illari, 238-54. New York: Routledge. 
Affolter, Markus, Savério Bellusci, Nobuyuki Itoh, Benny Shilo, Jean Paul Thiery, and Zena Werb. 2003. "Tube or Not Tube: Remodeling Epithelial Tissues by Branching Morphogenesis." Developmental Cell 4:11-18.

Affolter, Markus, Rolf Zeller, and Emmanuel Caussinus. 2009. “Tissue Remodelling through Branching Morphogenesis.” Nature Reviews Molecular Cell Biology 10: 831-42.

Bechtel, William. 2009. “Generalization and Discovery by Assuming Conserved Mechanisms: Cross-Species Research on Circadian Oscillators.” Philosophy of Science 76 (5): 762-73.

--------. 2017. “Explaining Features of Fine-Grained Phenomena Using Abstract Analyses of Phenomena and Mechanisms: Two Examples from Chronobiology.” Synthese, 1-23.

Bellusci, Savério, Justin Grindley, Hisayo Emoto, Nobuyuki Itoh, and Brigid L. M. Hogan. 1997. "Fibroblast Growth Factor 10 (FGF10) and Branching Morphogenesis in the Embryonic Mouse Lung.” Development 124: 4867-78.

Burian, Richard M., and Denis Thieffry. 2000. "Introduction to the Special Issue 'From Embryology to Developmental Biology.'” History and Philosophy of Life Sciences 22 (3): 313-23.

Davies, Jamie A. 2002. “Do Different Branching Epithelia Use a Conserved Developmental Mechanism?" BioEssays 24 (10): 937-48.

Fontana, Luigi, Linda Partridge, and Valter D. Longo. 2010. “Extending Healthy Life Span--From Yeast to Humans.” Science 328 (5976): 321-26.

Gelfert, Axel. 2013. "Strategies of Model-Building in Condensed Matter Physics: Trade-Offs as a Demarcation Criterion between Physics and Biology?" Synthese 190: 253-72.

Gerhardt, Holger, Matthew Golding, Marcus Fruttiger, Christiana Ruhrberg, Andrea Lundkvist, Alexandra Abramsson, Michael Jeltsch, et al. 2003. "VEGF Guides Angiogenic 
Sprouting Utilizing Endothelial Tip Cell Filopodia.” Journal of Cell Biology 161 (6): $1163-77$.

Ghabrial, Amin S., and Mark A. Krasnow. 2006. "Social Interactions among Epithelial Cells during Tracheal Branching Morphogenesis.” Nature 441: 746-49.

Gilbert, Scott F. 2014. Developmental Biology. 10th edition. Sunderland, MA: Sinauer Associates.

Glennan, Stuart S., and Phyllis Illari. 2018. "Introduction: Mechanisms and Mechanical Philosophies.” In The Routledge Handbook of Mechanisms and Mechanical Philosophy, edited by Stuart S. Glennan and Phyllis Illari, 1-9. New York: Routledge.

Halina, Marta, and William Bechtel. 2013. "Mechanism, Conserved.” In Encyclopedia of Systems Biology, edited by Werner Dubitzky, Olaf Wolkenhauer, Kwang-Hyun Cho, and Hiroki Yokota, 1201-4. New York: Springer.

Harmer, Stacey L, Satchidananda Panda, and Steve A. Kay. 2001. "Molecular Bases of Circadian Rhythms.” Annual Review of Cell and Developmental Biology 17: 215-53. Hellström, Mats, Li-Kun Phng, Jennifer J. Hofmann, Elisabet Wallgard, Leigh Coultas, Per Lindblom, Jackelyn Alva, et al. 2007. "D114 Signalling through Notch1 Regulates Formation of Tip Cells during Angiogenesis." Nature 1-445: 776-80.

Hopwood, Nick. 2009. "Embryology." In The Cambridge History of Science: The Modern Biological and Earth Sciences, edited by Peter J. Bowler and John V. Pickstone, 285315. Cambridge: Cambridge University Press.

Levins, Richard. 1966. "The Strategy of Model Building in Population Biology.” American Scientist 54 (4): 421-31. 
Levy, Arnon, and William Bechtel. 2013. "Abstraction and the Organization of Mechanisms." Philosophy of Science 80: 241-61.

Llimargas, Marta. 1999. "The Notch Pathway Helps to Pattern the Tips of the Drosophila Tracheal Branches by Selecting Cell Fates.” Development 126: 2355-64.

Love, Alan C. 2018. “Developmental Mechanisms.” In The Routledge Handbook of Mechanisms and Mechanical Philosophy, edited by Stuart S. Glennan and Phyllis Illari, 332-47. New York: Routledge.

Matthewson, John. 2011. "Trade-Offs in Model-Building: A More Target-Oriented Approach." Studies in History and Philosophy of Science Part A 42: 324-33.

--------. 2018. "Detail and Generality in Mechanistic Explanation.” Studies in History and Philosophy of Science, 1-9.

Matthewson, John, and Michael Weisberg. 2009. "The Structure of Tradeoffs in Model Building." Synthese 170: 169-90.

Metzger, Ross J., and Mark A. Krasnow. 1999. "Genetic Control of Branching Morphogenesis." Science 284: 1635-40.

Min, Hosung, Dimitry M. Danilenko, Sheila A. Scully, Brad Bolon, Brian D. Ring, John E. Tarpley, Margaret DeRose, and W. Scott Simonet. 1998. "Fgf-10 Is Required for Both Limb and Lung Development and Exhibits Striking Functional Similarity to Drosophila Branchless." Genes and Development 12: 3156-61.

Munóz-Chápuli R (2011) “Evolution of angiogenesis.” International Journal of Developmental Biology 55: 345-351.

Murray, Michael J. 2015. “Drosophila Models of Cancer.” AIMS Genetics 2 (1): 97-103. 
Nogawa, Hiroyuki, Kuniharu Morita, and Wellington V. Cardoso. 1998. "Bud Formation

Precedes the Appearance of Differential Cell Proliferation during Branching Morphogenesis of Mouse Lung Epithelium in Vitro.” Developmental Dynamics 213: $228-35$.

Ochoa-Espinosa, Amanda, and Markus Affolter. 2012. "Branching Morphogenesis: From Cells to Organs and Back." Cold Spring Harbor Perspectives in Biology 4: 1-14.

Odenbaugh, Jay. 2003. “Complex Systems, Trade-offs, and Theoretical Population Biology: Richard Levin’s ‘Strategy of Model Building in Bopulation Biology’ Revisited.” Philosophy of Science 70: 1496-1507.

Orzack, Steven Hecht, and Elliott Sober. 1993. “A Critical Assessment of Levins's The Strategy of Model Building in Population Biology (1966)." The Quarterly Review of Biology 68 (4): $533-46$.

Park, William Y., Barbara Miranda, Djamel Lebeche, Gakuji Hashimoto, and Wellington V. Cardoso. 1998. "FGF-10 Is a Chemotactic Factor for Distal Epithelial Buds during Lung Development.” Developmental Biology 201: 125-34.

Ryan, Tomás J, and Seth G. N. Grant. 2009. “The Origin and Evolution of Synapses.” Nature Reviews Neuroscience 10: 701-12.

Sekine, Keisuke, Hideyo Ohuchi, Masanori Fujiwara, Masahiro Yamasaki, Tatsuya Yoshizawa, Takashi Sato, Naoko Yagishita, et al. 1999. "Fgf10 Is Essential for Limb and Lung Formation." Nature Genetics 21: 138-41.

Sheredos, Benjamin, Daniel Burnston, Adele Abrahamsen, and William Bechtel. 2013. "Why Do Biologists Use so Many Diagrams?” Philosophy of Science 80 (5): 931-44. 
Siekmann, Arndt F., and Nathan D. Lawson. 2007. "Notch Signalling Limits Angiogenic Cell Behaviour in Developing Zebrafish Arteries." Nature 445: 781-84.

Siekmann, Arndt F., Laurence Covassin, and Nathan D. Lawson. 2008. "Modulation of VEGF Signalling Output by the Notch Pathway.” BioEssays 30 (4): 303-13.

Spurlin, James W., and Celeste M. Nelson. 2017. "Building Branched Tissue Structures: From Single Cell Guidance to Coordinated Construction." Philosophical Transactions of the Royal Society B: Biological Sciences 372: 1-15.

Sutherland, David, Christos Samakovlis, and Mark A. Krasnow. 1996. "branchless Encodes a Drosophila FGF Homolog That Controls Tracheal Cell Migration and the Pattern of Branching." Cell 87: 1091-1101.

Trinkaus, John P. 1969. Cells into Organs: The Forces That Shape the Embryo. Englewood Cliffs: Prentice Hall.

Varner, Victor D., and Celeste M. Nelson. 2014. "Cellular and Physical Mechanisms of Branching Morphogenesis.” Development 141: 2750-59.

Waddington, Conrad Hal. 1956. Principles of Embryology. New York: The Macmillan Co. Wang, Shaohe, Rei Sekiguchi, William P. Daley, and Kenneth M. Yamada. 2017. "Patterned Cell and Matrix Dynamics in Branching Morphogenesis.” The Journal of Cell Biology, $1-12$.

Waters, C. Kenneth. 1998. "Causal Regularities in the Biological World of Contingent Distribution.” Biology and Philosophy 13: 5-36.

Weisberg, Michael. 2004. “Qualitative Theory and Chemical Explanation.” Philosophy of Science 71: 1071-81.

--------. 2006. “Robustness Analysis.” Philosophy of Science 73: 730-42. 
--------. 2007. “Three Kinds of Idealization.” The Journal of Philosophy 104 (12): 639-59. 2013. Simulation and Similarity: Using Models to Understand the World. Oxford:

Oxford University Press.

Wimsatt, William C. 2012. "Robustness, Reliablity, and Overdetermination (1981).” In

Characterizing the Robustness of Science, edited by Léna Soler, Emiliano Trizio, Thomas Nickles, and William C Wimsatt, 61-87. Dordrecht: Springer.

Woody, Andrea I.2004. "More Telltale Signs: What Attention to Representation Reveals about Scientific Explanation.” Philosophy of Science 71: 780-93.

2014. "Chemistry's Periodic Law: Rethinking Representation and Explanation after the Turn to Practice." In Science after the Practice Turn in the Philosophy, History, and the Social Studies of Science, edited by Léna Soler, Sjoerd Zwart, Michael Lynch, and Vincent Israel-Jost, 123-50. New York: Routledge.

--------. 2015. "Re-Orienting Discussions of Scientific Explanation: A Functional Perspective." Studies in History and Philosophy of Science Part A 52: 79-87. 

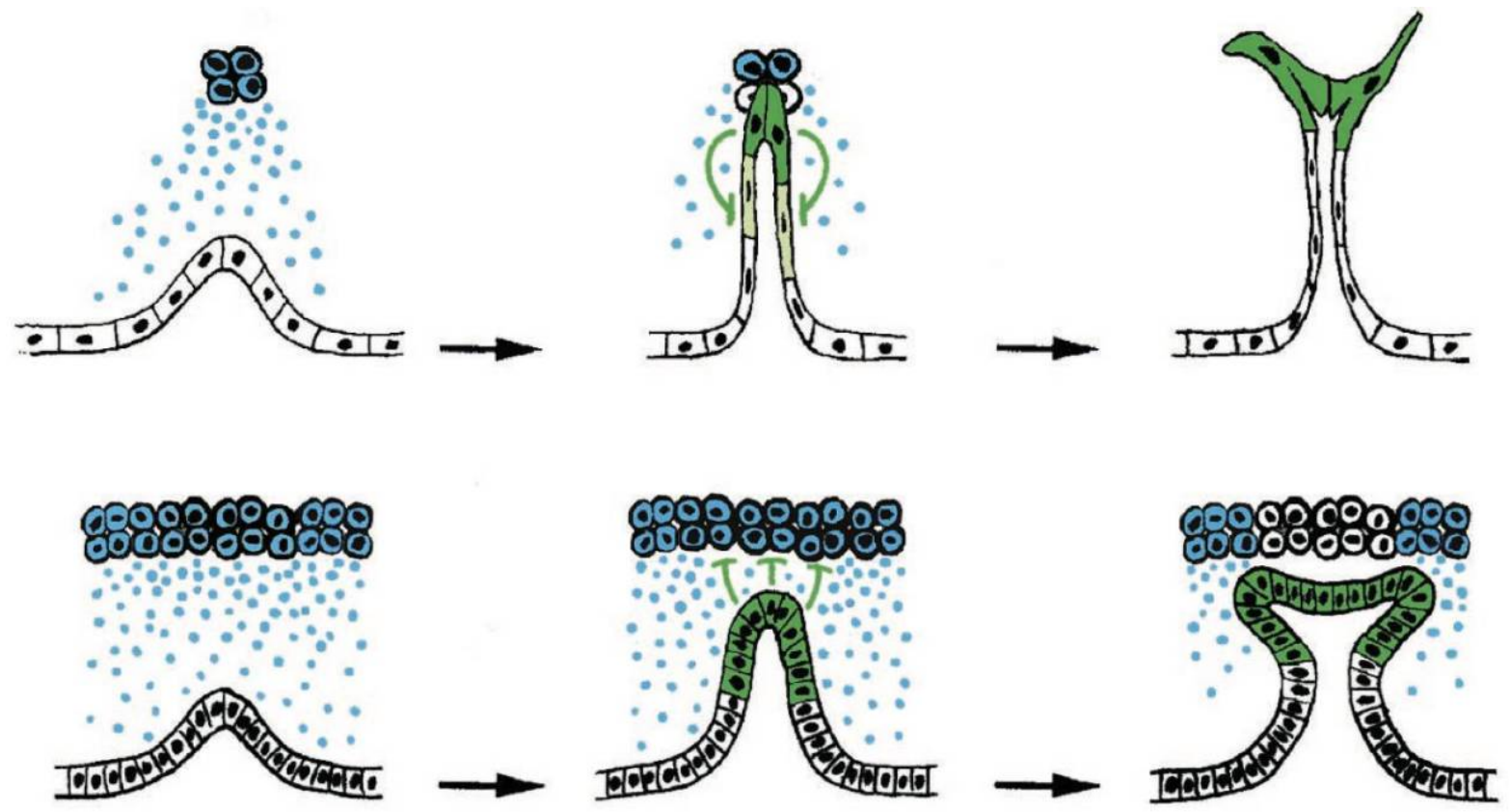

Figure1. Part of figure 2 of Metzger and Krasnow (1999), where the models of fly tracheal formation (top) and mammalian lung formation (bottom) are displayed together. The commonality between the two mechanisms is highlighted by the use of the same colors (blue: FGF ligand and the cells that secrete it; green: cells at the leading tips of the branches extending towards the sources of FGF). Differences (the activity of the epithelial cells and the overall organization) are also indicated visually. Reprinted with permission of the American Association for the Advancement of Science. Color version available as an online enhancement. 
A

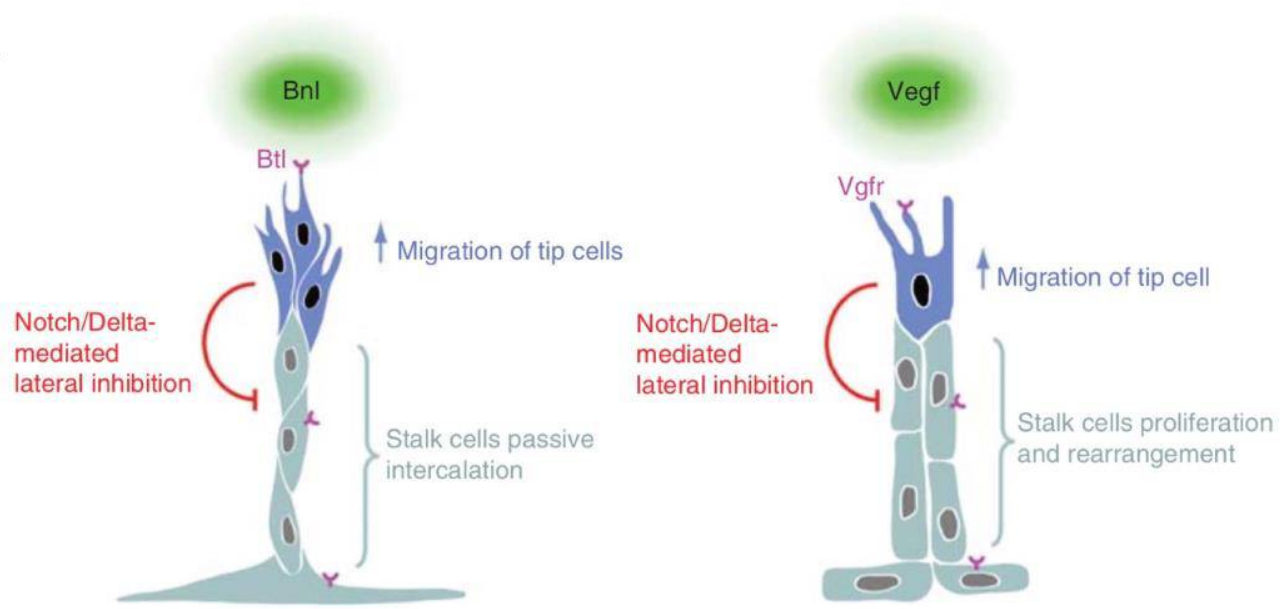

B
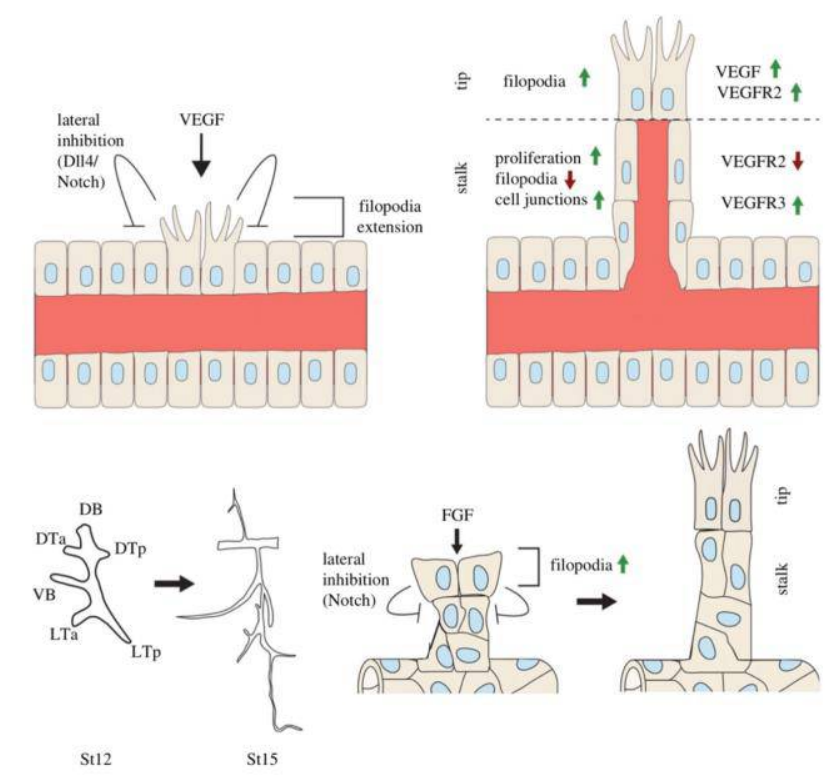

C

"Budding" in Drosophila trachea

High FGFR/Btl FGF/Bnl

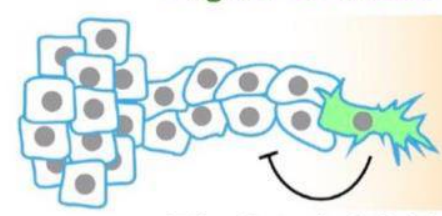

Tip fate inhibition

by Delta/Notch
"Budding" in blood vessels

VEGFR VEGF

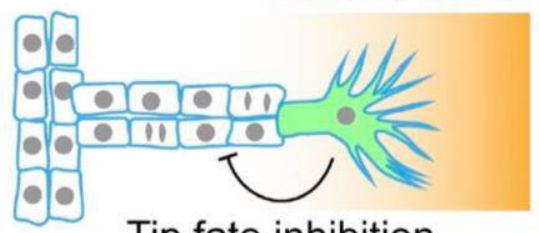

Tip fate inhibition

by Delta/Notch 
Figure 2. Examples of figures where the mechanistic models of fly tracheal formation and vertebrate angiogenesis are displayed together. Commonalities (roles of tip and stalk cells and interaction between them) are highlighted by the use of text, colors, and flat-edged arrows. Differences (types of the diffusing signaling molecules and activities of stalk cells) are also indicated visually and textually. (A) Figure 2 of Ochoa-Espinosa and Affolter (2012). Left: fly tracheal formation; right: vertebrate angiogenesis. Reprinted with permission of Cold Spring Harbor Laboratory Press. (B) Figure 3 of Spurlin and Nelson (2017). Top: vertebrate angiogenesis; bottom: fly tracheal formation. Reprinted with permission of Royal Society Publishing. (C) Part of figure 2 of Wang et al. (2017). Reprinted with permission of The Rockefeller University Press. Color version available as an online enhancement. 
TABLE 1. COMPARISON BETWEEN MMI AND MMJ.

\begin{tabular}{lll}
\hline How to manage trade-offs? & $\begin{array}{l}\text { MMI } \\
\text { Different models exhibit } \\
\text { different desiderata }\end{array}$ & $\begin{array}{l}\text { MMJ } \\
\text { Individual models exhibit } \\
\text { certain desiderata, while other } \\
\text { desiderata are fulfilled by a } \\
\text { comparison of those models }\end{array}$ \\
Types of models & Different & Same \\
Comparison of models & $\begin{array}{l}\text { Inessential; can be used } \\
\text { for robustness analysis }\end{array}$ & $\begin{array}{l}\text { Essential; to fulfill } \\
\text { distinct desiderata }\end{array}$ \\
$\begin{array}{l}\text { Purpose of using } \\
\text { multiple models }\end{array}$ & $\begin{array}{l}\text { To account for a single } \\
\text { phenomenon from } \\
\text { different perspectives }\end{array}$ & $\begin{array}{l}\text { To compare distinct } \\
\text { phenomena by comparing } \\
\text { models of them }\end{array}$ \\
\hline
\end{tabular}



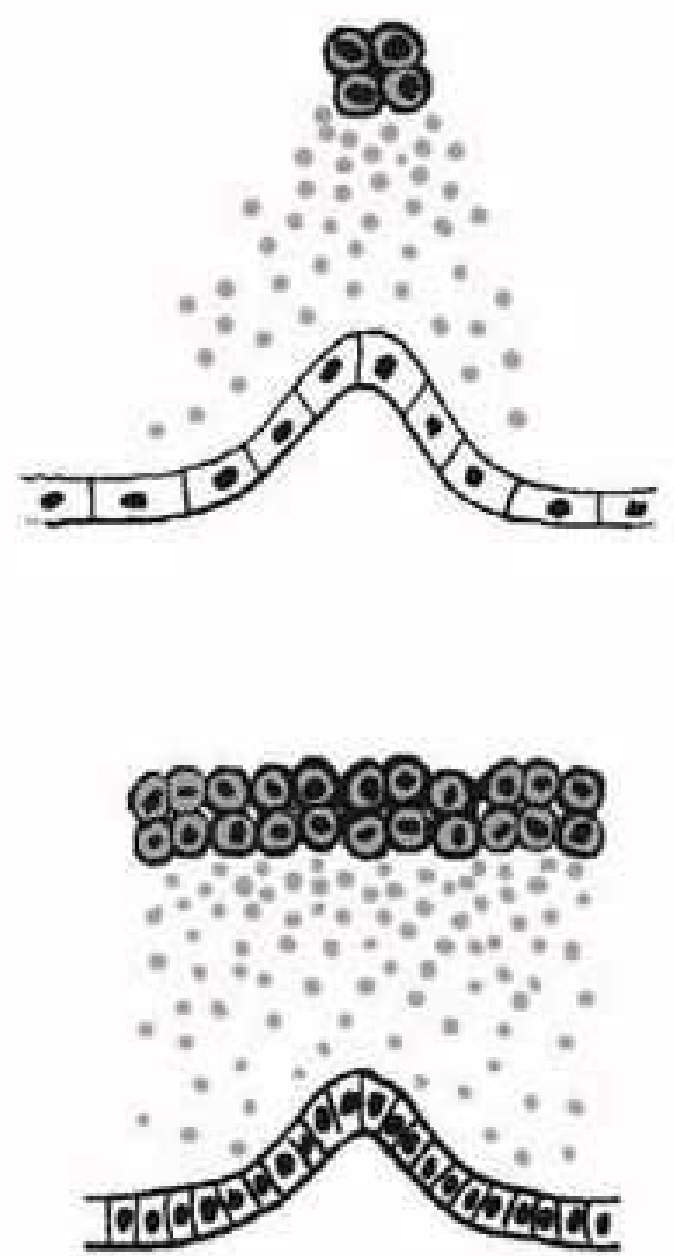
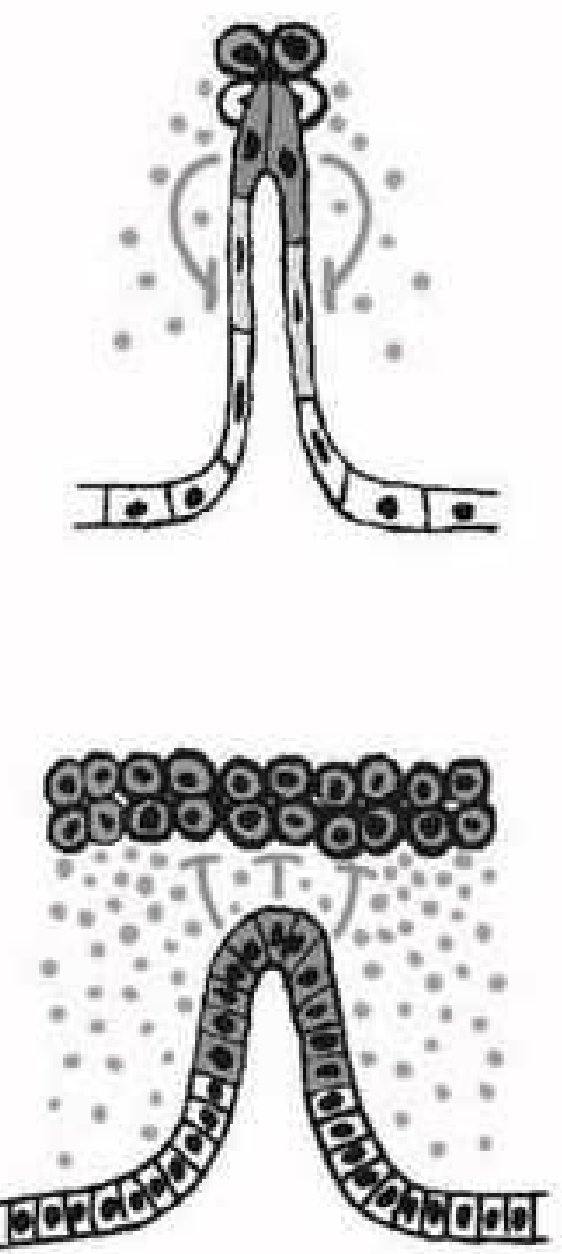
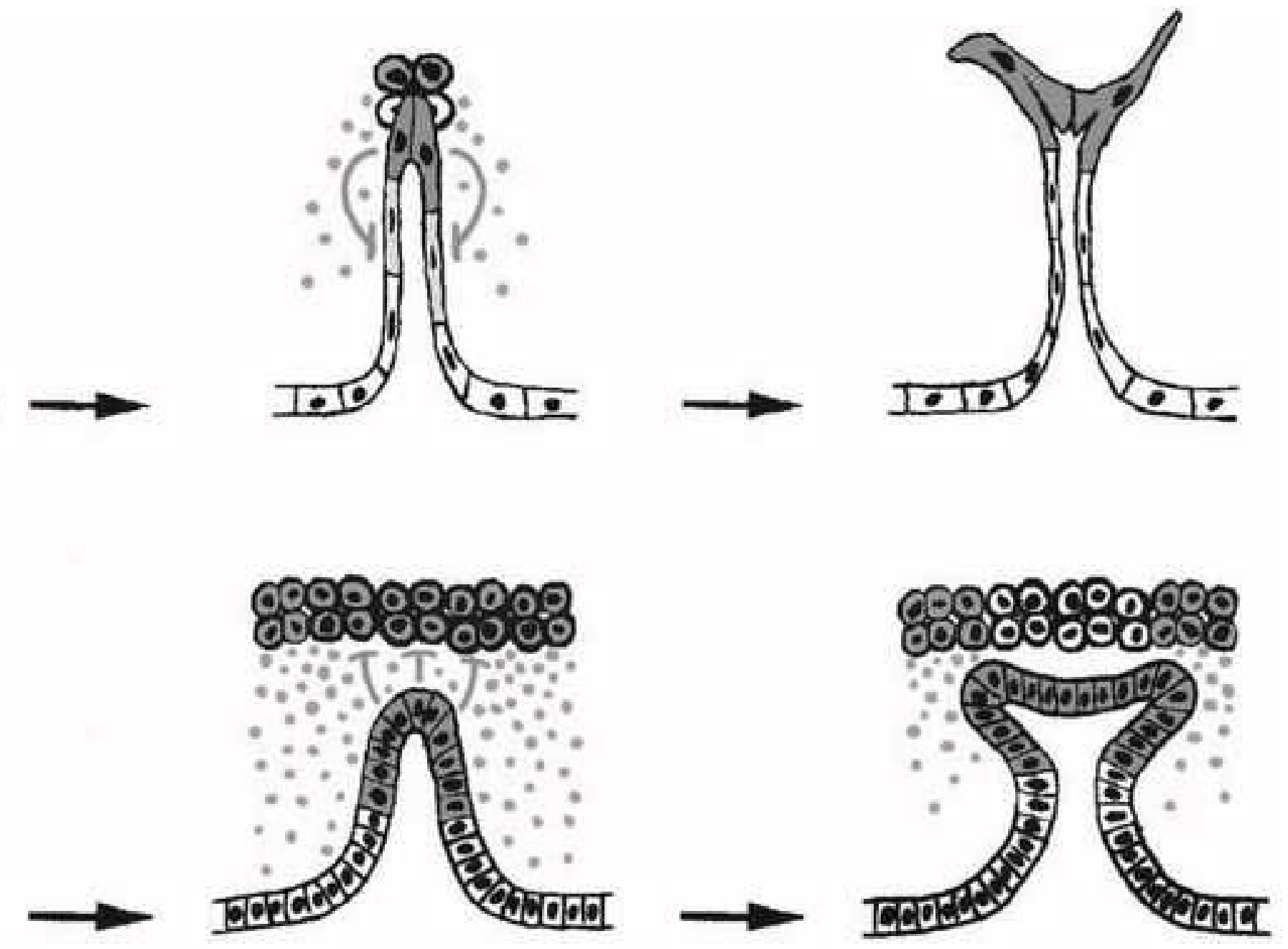
Click here to access/download;Figure;Juxtaposition_fig2_bw.jpg $\stackrel{\underline{ }}{ }$

Copyright The Philosophy of Science Association 2020.

Preprint (not copyedited or formatted).

Please use DOI when citing or quoting. DOI: https://doi.org/10.1086/710054.

A

Bnt

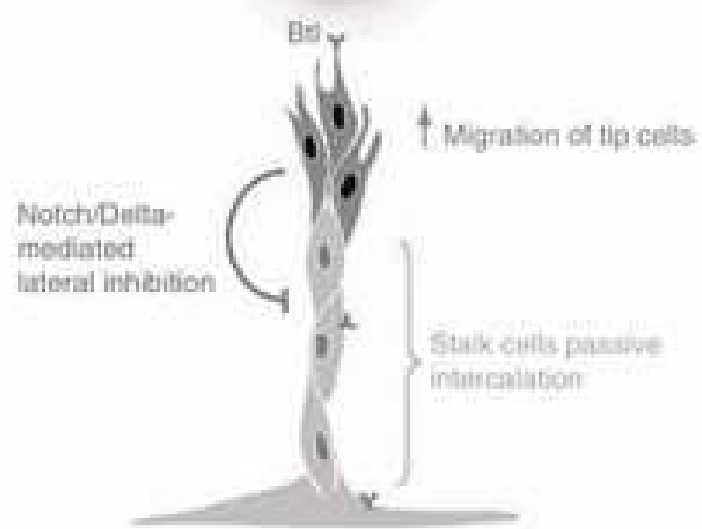

Vegt

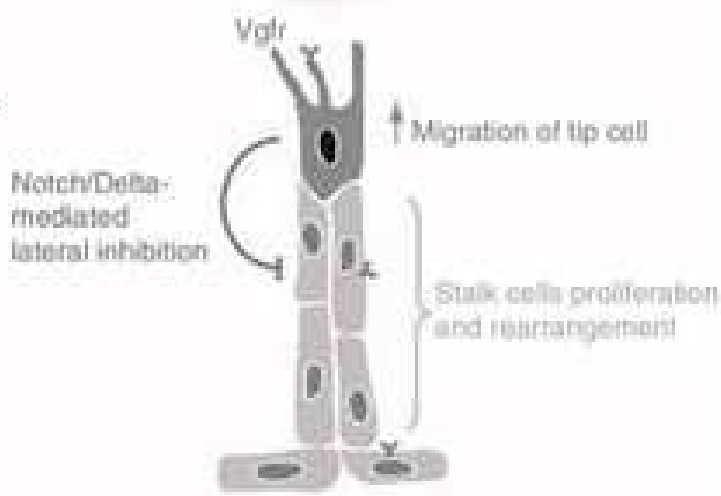

B
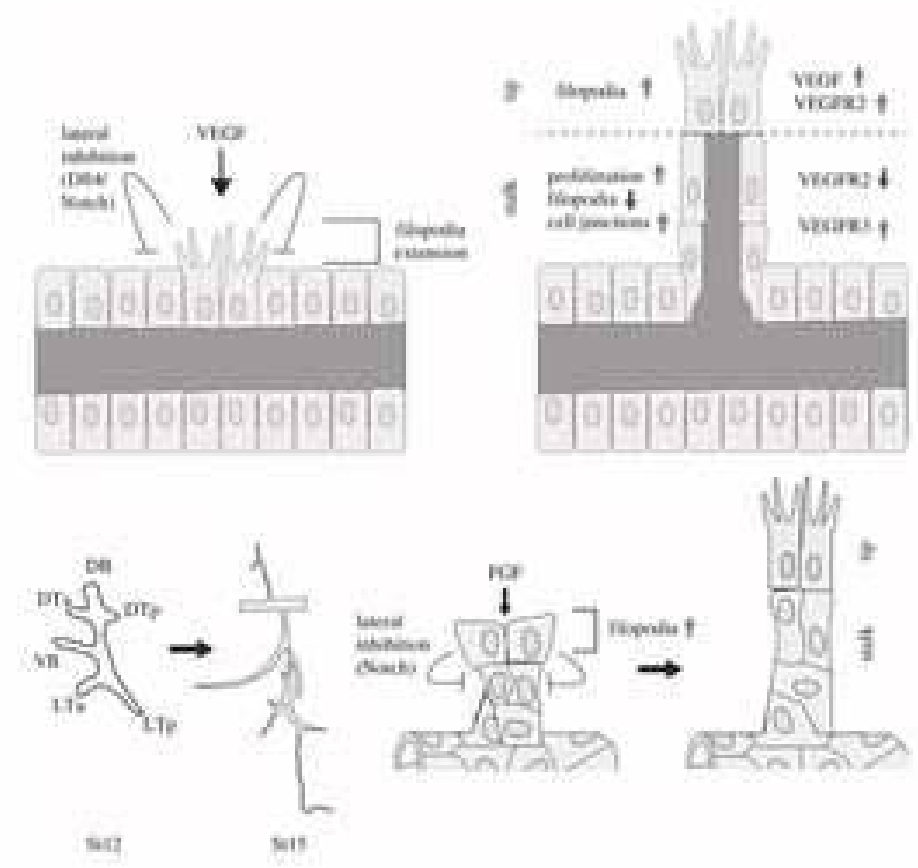

C

"Budding" in Drosophila trachea High FGFR/Btl FGF/Bnl

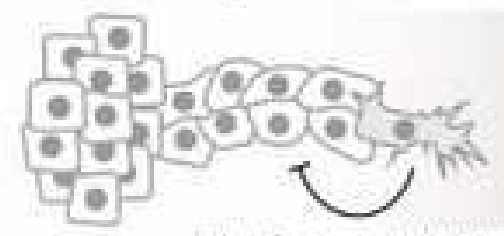

Tip fate inhibition by Delta/Notch
"Budding" in blood vessels

VEGFR VEGF

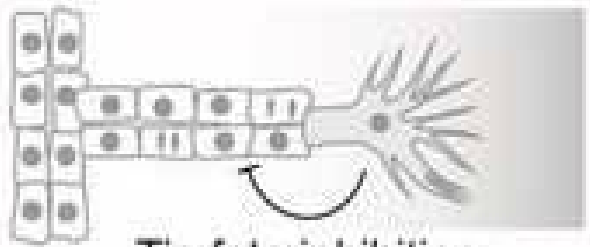

Tip fate inhibition by Delta/Notch 